\title{
Sechs Jahre HMD Best Paper Award - Zwischenbilanz und Ausblick
}

\author{
Matthias Knoll • Sybille Thelen
}

Online publiziert: 27. März 2017

C) Springer Fachmedien Wiesbaden 2017

Seit nunmehr über einem halben Jahrhundert und über 300 Ausgaben gehen Jahr für Jahr zahlreiche interessante Beiträge bei uns, den Herausgeberinnen und Herausgebern der HMD, ein. Sei es im Rahmen von Schwerpunktbeiträgen oder als Beitrag zu anderen aktuellen Themen der Wirtschaftsinformatik. In den Herausgebersitzungen wurde mancher Beitrag lobend herausgehoben, noch einmal näher betrachtet und kommentiert. Warum, so unser Gedanke Ende 2011, sollten wir diese Überlegungen, Diskussionen und Einschätzungen für uns behalten? Warum nicht einen Best Paper Award verleihen?

Mit dem „HMD Best Paper Award“ werden nun also bereits im sechsten Jahr alljährlich die drei besten Beiträge eines Jahrgangs der Zeitschrift „HMD - Praxis der Wirtschaftsinformatik“ gewürdigt. Die Auswahl der Beiträge erfolgt durch das HMD-Herausgebergremium und orientiert sich an folgenden Kriterien:

- Zielgruppenadressierung

- Handlungsorientierung und Nachhaltigkeit

- Originalität und Neuigkeitsgehalt

- Erkennbarer Beitrag zum Erkenntnisfortschritt

- Nachvollziehbarkeit und Überzeugungskraft

- Sprachliche Lesbarkeit und Lebendigkeit

Den allerersten HMD Best Paper Award verlieh unsere damalige Schriftleiterin Prof. Susanne Strahringer (TU Dresden) für das Jahr 2011 in Braunschweig auf der dortigen Multikonferenz Wirtschaftsinformatik (MKWI) 2012. Unser Ziel war und

\footnotetext{
M. Knoll ( $\bowtie)$

Hochschule Darmstadt, Darmstadt, Deutschland

E-Mail: matthias.knoll@h-da.de

S. Thelen

Springer Vieweg, Wiesbaden, Deutschland

E-Mail: sybille.thelen@springer.com
} 
ist es, unseren Lesern und auch solchen, die die „HMD - Praxis der Wirtschaftsinformatik“ noch nicht kennen, mit dem „HMD Best Paper Award“ eine kleine Sammlung an Beiträgen an die zu Hand geben, die wir für besonders lesenswert halten, und den Autoren, denen wir diese Beiträge zu verdanken haben, damit zugleich unsere besondere Anerkennung zu zeigen. Alle Preisträger erhalten im Rahmen der Preisverleihung im Frühjahr des Folgejahres eine individuelle Urkunde. Seit drei Jahren, beginnend mit den Best Papern von 2013, werden die Beiträge auch in der „Essentials“-Reihe von Springer im Originalformat oder einer überarbeiteten, meist ergänzten Fassung veröffentlicht.

Für das Jahr 2016 wurden im Rahmen der WI 2017 in St. Gallen die folgenden Beiträge prämiert:

- Brandes, C./Heller, M.: Qualitätsmanagement in agilen Projekten - quo vadis? HMD - Praxis der Wirtschaftsinformatik 53 (2016), 308, S. 169-184.

Auch mit der Umsetzung neuartiger Vorgehensweisen und Methoden wird Qualitätsmanagement in der IT heute immer noch als „Achillesferse“ wahrgenommen. Die Stimmen, die sich darüber beklagen, dass die IT nicht die von den Kunden bzw. Endbenutzern erwarteten Lösungen liefert, sind nicht zu überhören. Der Beitrag löst anhand praktischer Projektbeispiele hartnäckige Missverständnisse zwischen „Qualität““ und „Agilität“ auf und zeigt konkrete Handlungsmöglichkeiten, wie auch im Qualitätsmanagement die Vorteile agiler Vorgehensweisen nutzbar gemacht werden können.

- Böck, M./Köbler, F./Anderl, E./Le, L.: Social Media-Analyse - Mehr als nur eine Wordcloud? HMD - Praxis der Wirtschaftsinformatik 53 (2016), 309, S. 323-338. Die Analyse von Social Mediadaten ist ein zunehmend wichtigeres Forschungsfeld. Anhand von konkreten Anwendungen werden im Beitrag wichtige aktuelle Auswertungsverfahren beschrieben. Neben deskriptiven Auswertungen beleuchtet er den Nutzen von Sentimentanalysen, Assoziationsregeln und Graphalgorithmen. Spannend dabei ist, dass das Sample aus Twitter- und Facebook-Daten zum Thema „Tatort“ stammt. Der Datensatz enthält eine halbe Million Einträge von fast hunderttausend Nutzern, wobei die in deutscher Sprache verfassten Nachrichten herausgefiltert, in einer NoSQL-Datenbank gespeichert und mit der Programmiersprache R für statistische Auswertungen analysiert wurden.

- Schröder, H./Müller, A.: Szenarien und Vorgehen für die Gestaltung der IT-Organisation von morgen. HMD - Praxis der Wirtschaftsinformatik 53 (2016), 311, S. 580-593.

Effiziente Informationshaltung mit beliebiger Skalierbarkeit benötigter Ressourcen und ubiquitärer Verfügbarkeit auf mobilen Medien ist ein Erfolgskriterium für modernes IT-Management. Neben Infrastructure as a Service und Platform as a Service setzt sich zunehmend auch Software as a Service durch - auf privaten, öffentlichen oder hybriden Cloud-Systemen.

Der Beitrag stellt sehr anschaulich die neu entstehenden Anforderungen an die IT-Organisation dar, die der Einsatz von Cloud Computing für die Unternehmen mit sich bringt. Er fokussiert hierbei auf ein 3-stufiges Phasenmodell zur Gestaltung einer zukunftsfähigen IT-Organisation, die eine auf die Bedürfnisse des Cloud Computing angepasste Aufgabenverteilung zwischen der IT-Abteilung, den Fachabteilungen und externen Partnern unternehmensspezifisch zum Ziel hat. 


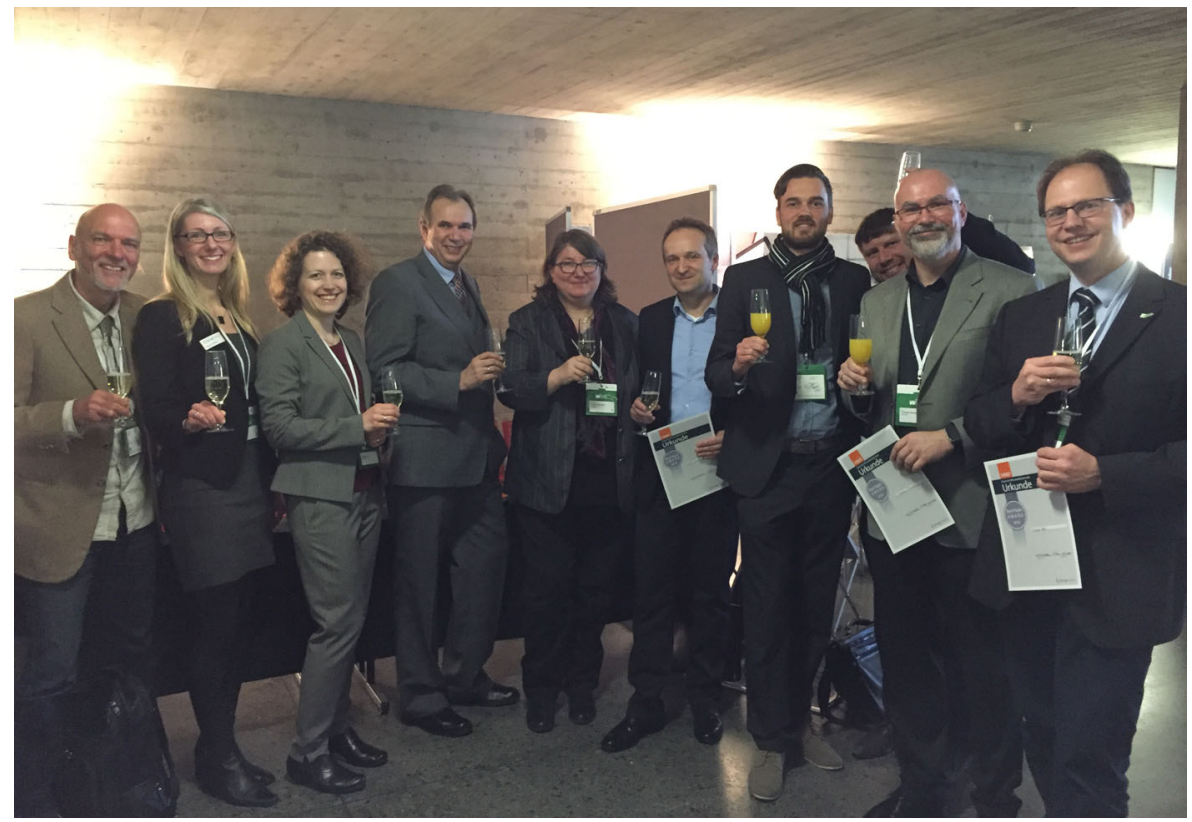

Abb. 1 Verleihung HMD Best Paper Award: (v.l.n.r.) A. Meier, S. Kathke, S. Thelen, A. Müller, S. Strahringer, H. Schröder, F. Köbler, D. Zumstein, C. Brandes, M. Heller

Alle drei prämierten Beiträge haben sich in mehreren der genannten Kriterien von den anderen Beiträgen abgesetzt und verdienen daher besondere Aufmerksamkeit.

Es hat uns sehr gefreut, dass sich alle Preisträger Zeit genommen und den Weg nach St. Gallen gefunden haben. Nach der feierlichen Verleihung und Überreichung der Urkunden nutzten Preisträger, Herausgeber und Gäste die angenehme Atmosphäre zum Netzwerken bei guten Gesprächen (siehe Abb. 1).

Die Gewinner der Vorjahre (in der Reihenfolge der Veröffentlichung in den jeweiligen HMD-Ausgaben):

- 2011

- T. Goetz, P. Maurer, Musterbasierte Anwendungsintegration, HMD 278

- P. Helmholtz, M. Böringer, S. Robra-Bissantz, Meinungen und Prognosen in Twitter - das Ohr an der Masse, HMD 280

- M. Ardelt, F. Dölitzscher, M. Knahl, C. Reich, Sicherheitsprobleme für ITOutsourcing durch Cloud Computing, HMD 281

- 2012

- M. Wiener, R. Denk, Governance von globalen IT-Projekten - eine dynamische Kontrollperspektive, HMD 284

- E. Pergler, C. Adelsberger, C. Kittl, M. Altenstrasser, Die mobile Kundenkarte, HMD 286

- S. Zimmermann, C. Rentrop, Schatten-IT, HMD 288 
- 2013

- A. Wiedenhofer, Flexibilitätspotenziale heben, HMD 289. Essentials: ISBN print 978-3-658-06710-6; ISBN eBook 978-3-658-06711-3

- G. Disterer, C. Kleiner, Mobile Endgeräte im Unternehmen, HMD 290. Essentials: ISBN print 978-3-658-07023-6; ISBN eBook 978-3-658-07024-3

- N. Pelzl, A. Helferich, G. Herzwurm, Wertschöpfungsnetzwerke dt. CloudAnbieter, HMD 292. Essentials: ISBN print 978-3-658-07010-6; ISBN eBook 978-3-658-07011-3

- 2014

- T. Walter, Bring your own Device, HMD 295. Essentials: ISBN print 978-3658-11590-6; ISBN eBook 978-3-658-11591-3

- S. Wachter, T. Zaelke, Systemkonsolidierung und Datenmigration, HMD 296. Essentials: ISBN print 978-3-658-11405-3; ISBN eBook 978-3-658-11406-0

- A. Györy, G. Seeser, A. Cleven, F. Uebernickel, W. Brenner, Projektübergreifendes Applikationsmanagement, HMD 299. Essentials: ISBN print 978-3658-12328-4; ISBN eBook 978-3-658-12329-1

- 2015

- P. Lotz, E-Commerce und Datenschutzrecht im Konflikt, HMD 302. Essentials: ISBN print 978-3-658-14160-8; ISBN eBook 978-3-658-14161-5

- M. Herterich, F. Uebernickel, W. Brenner, Industrielle Dienstleistungen 4.0, HMD 305. Essentials: ISBN print 978-3-658-13910-0; ISBN eBook 978-3658-13911-7

- S. Schacht, A. Reindl, S. Morana, A. Mädche, Projektwissen spielend einfach managen mit der ProjectWorld, HMD 306. Essentials: ISBN print 978-3-65814853-9; ISBN eBook 978-3-658-14854-6

Die HMD blickt auf eine sehr lange Geschichte und Tradition zurück: Im Oktober 1964 wurde das Grundwerk der ursprünglichen Loseblattsammlung unter dem Namen „Handbuch der maschinellen Datenverarbeitung“ ausgeliefert. Seit 1998 lautet der Titel der Zeitschrift unter Beibehaltung des bekannten HMD-Logos „Praxis der Wirtschaftsinformatik“, seit Januar 2014 erscheint sie bei Springer Vieweg. Alle HMD-Beiträge basieren damals wie heute auf einem Transfer wissenschaftlicher Erkenntnisse in die Praxis der Wirtschaftsinformatik. Umfangreiche Themengebiete werden in HMD-Heften aus verschiedenen Blickwinkeln betrachtet, so dass in jedem Heft sowohl Wissenschaftler als auch Praktiker zu einem aktuellen Schwerpunktthema zu Wort kommen. Den verschiedenen Facetten eines Schwerpunktthemas geht ein Grundlagenbeitrag zum State of the Art des Themenbereichs voraus. Damit liefert die HMD IT-Fach- und Führungskräften Lösungsideen für ihre Probleme, zeigt ihnen Umsetzungsmöglichkeiten auf und informiert sie über Neues in der Wirtschaftsinformatik. Studierende und Lehrende der Wirtschaftsinformatik erfahren zudem, welche Themen in der Praxis ihres Faches Herausforderungen darstellen und aktuell diskutiert werden.

So wie wir die Tradition der HMD - Praxis der Wirtschaftsinformatik fortsetzen, möchten wir auch den HMD Best Paper Award zu einer Tradition werden lassen. Wir sind sicher, dass uns die exzellenten Beiträge auch künftig nicht ausgehen werden - und möchten Sie ermuntern, rege Beiträge einzureichen, auch wenn wir es dann 
gewiss nicht leichter haben werden, aus der Vielzahl der guten Einreichungen die besten drei auszuwählen.

In diesem Sinne freuen wir uns bereits jetzt auf Ihre Beiträge in den Heften des aktuellen Jahres und die Preisverleihung auf der MKWI in Lüneburg im März 2018 sowie die damit verbundene schöne und ehrenvolle Aufgabe der Kür der besten Beiträge 2017!

Die Herausgeberinnen und Herausgeber der HMD und der Verlag 